\title{
Probability distribution of energetic-statistical size effect in quasibrittle fracture
}

\author{
Zdeněk P. Bažant* \\ Department of Civil and Environmental Engineering, McCormick School of Engineering and Applied Science, \\ Northwestern University, 2145 Sheridan Road, Evanston, IL 60208-3109, USA
}

Received 30 June 2003; revised 1 August 2003; accepted 4 September 2003

\begin{abstract}
The physical sources of randomness in quasibrittle fracture described by the cohesive crack model are discussed and theoretical arguments for the basic form of the probability distribution are presented. The probability distribution of the size effect on the nominal strength of structures made of heterogeneous quasibrittle materials is derived, under certain simplifying assumptions, from the nonlocal generalization of Weibull theory. Attention is limited to structures of positive geometry failing at the initiation of macroscopic crack growth from a zone of distributed cracking. It is shown that, for small structures, which do not dwarf the fracture process zone (FPZ), the mean size effect is deterministic, agreeing with the energetic size effect theory, which describes the size effect due to stress redistribution and the associated energy release caused by finite size of the FPZ formed before failure. Material randomness governs the statistical distribution of the nominal strength of structure and, for very large structure sizes, also the mean. The large-size and small-size asymptotic properties of size effect are determined, and the reasons for the existence of intermediate asymptotics are pointed out. Asymptotic matching is then used to obtain an approximate closed-form analytical expression for the probability distribution of failure load for any structure size. For large sizes, the probability distribution converges to the Weibull distribution for the weakest link model, and for small sizes, it converges to the Gaussian distribution justified by Daniels' fiber bundle model. Comparisons with experimental data on the size-dependence of the modulus of rupture of concrete and laminates are shown. Monte Carlo simulations with finite elements are the subject of ongoing studies by Pang at Northwestern University to be reported later.
\end{abstract}

(C) 2003 Elsevier Ltd. All rights reserved.

Keywords: Probability distribution; Size effect; Quasibrittle materials; Fracture mechanics; Nonlocal continuum; Failure; Extreme value statistics; Weakest link model; Asymptotic analysis

\section{Introduction}

With the recent surge of interest in scaling and scalebridging, the size effect in solid mechanics came to the forefront of attention. Until the late 1980s, it was widely believed that any experimentally observed size effect on the nominal strength of structures was of statistical origin, caused by randomness of local material strength and described by Weibull statistical theory. However, beginning with the mid 1970s, it gradually transpired that there exists another type of size effect which is purely energetic by origin.

The energetic size effect, which is observed in heterogeneous quasibrittle materials such as concrete, fiber

\footnotetext{
* Tel.: + 1-847-491-4025; fax: + 1-847-467-4011.

E-mail address: z-bazant@northwestern.edu (Z.P. Bažant).
}

composites, rocks, tough ceramics, sea ice, dry snow slabs, wood, paper and some biomaterials, is caused by the fact that quasibrittle cohesive fracture is preceded by the formation of a fracture process zone (FPZ) that is not negligible compared to the cross section dimensions and is large enough to cause significant stress redistribution in a structure. The stress redistribution causes a significant energy release from the structure, which engenders an energetic size effect, i.e. a size dependence of the nominal (or apparent) strength of structure [1,2].

Because the local strength of a quasibrittle material is a random field, probabilistic aspects must play at least some role in the energetic size effect. Even when they do not control the mean size effect, they must be expected to dominate the variance and especially the extreme value distribution, the knowledge of which is essential for reliability assessments of structures. To capture the extreme 
value statistics of the energetic size effect in quasibrittle materials is the objective of the new model presented in this study.

\section{Types of energetic size effect}

Two basic types of energetic size effect may be distinguished [2-5]. Type 1 size effect [6-8] occurs in structures of positive geometry having no notches or preexisting large cracks. ${ }^{1}$ In such structures, the maximum load is reached as soon as a macroscopic crack initiates from the FPZ, which is as soon as the FPZ (a zone of microcracking) has fully formed. Tension and flexure of unnotched and unreinforced beam or plate are generally positive geometries. Note also that positive geometry is one of the requirements for the validity of Weibull weakest link model.

Type 2 size effect $[2,9,10]$ also occurs for positive geometry but the structure has notches, as in fracture specimens, or large stress-free cracks that have grown in a stable manner prior to the maximum load. This type of size effect, the mean of which is not significantly affected by material randomness [2,51], has been demonstrated and analyzed for fiber composites, both in tension [11] and compression [12], and will not be investigated here. Note that one can also distinguish a third type of size effect, which occurs when the geometry is initially negative and later changes to positive [2,4]. However, this third type of size effect is very similar to type 2 .

In this paper (the summary of which was presented at a recent conference [50]), only the size effect of type 1 will be treated. It appears to be the only type, where material randomness can influence the mean size effect significantly.

\section{Avenues towards general probabilistic-energetic size effect theory}

Traditionally the size effect has been explained by Weibull's statistical weakest link model [13-21,55]. Its basic hypothesis is that the structure fails as soon as the material strength is exhausted at one point of the structure. But this is far from true for quasibrittle materials.

To cope with quasibrittle failures, two avenues of research, aiming from opposite sides of the problem toward the same objective, have been pursued. One, which is the more classical one, is a generalization of the extreme value statistics of the weakest link mode by introduction of various phenomenological load-sharing hypotheses, the simplest prototype of which is Daniels' [22] fiber bundle model. In a way, these load-sharing hypotheses act similarly

\footnotetext{
${ }^{1}$ Structures of positive geometry are those in which the stress intensity factor, or the energy release rate, increases if the crack extends at constant load.
}

as the energy release due to stress redistribution by advancing fracture, but are far from equivalent. This avenue of approach, pursued by Phoenix and co-workers [23-32] has proven to be mathematically very challenging and has led to high mathematical sophistication. Important mathematical results have been achieved for the statistical distribution of strength in tensioned parallel structural systems such as ropes (or cables) consisting of fibers (or wires) obeying Weibull statistical distributions of strength.

However, this rigorous mathematical approach has not made it possible to deal with load-sharing of varying intensities among a very large number of fibers (or wires), nor with load-sharing properties governed by cohesive fracture mechanics. It is not clear how various load-sharing concepts could be generalized to two-dimensional stress redistribution by fracture and its FPZ, and how they could capture the disparity in energy release and energy dissipation rates, which is the physical source of energetic size effect-namely the fact the energy release rate grows with increasing structure size roughly quadratically while the energy dissipation rate grows roughly linearly. In summary, expanding the probabilistic load-sharing concepts for parallel systems to capture the salient properties of quasibrittle cohesive fracture in two or three dimensions appears to be a very difficult target.

Therefore, the problem is approached in this paper from the opposite side, as a probabilistic generalization of the energetic size effect theory. This is another, more recent, avenue of approach, which attempts to generalize the deterministic theory based on cohesive fracture mechanics such that deterministic size effect would be preserved as the mean. This avenue originated with the nonlocal generalization of Weibull theory [51, 33-35], which allowed statistical numerical simulations of the mean deterministic-statistical size effect and its variance. With the use of the technique of asymptotic matching [36-38], this led to a simple explicit formula for size effect, which provided a distinctly better agreement with the mean trend of the statistical size effect data on the modulus of rupture of concrete and laminates than the classical Weibull theory.

The goal of the present paper is to extend this theory to formulate a realistic approximation of the statistical distribution of the nominal strength of unnotched structures of positive geometry, and the dependence of this distribution on structure size. Verification of the proposed theory by Monte Carlo finite element simulations and by thorough studies of experimental evidence for fiber composites and concrete is left for a subsequent paper.

\section{Failure probability as a function of structure size (and geometry)}

The nonlocal generalization of Weibull statistical theory led to an energetic-statistical theory of size effect [51], which was shown to provide a good numerical description 
of the mean size effect of type 1 in concrete and fibercomposite laminates $[2,33-35,39,40,42]$. A simple formula for this size effect has been derived by asymptotic matching of the large-size limiting properties of linear elastic fracture mechanics and the small-size limiting properties nonlocal damage theory or cohesive crack model. Here, we will try to advance beyond the mean behavior and deduce a complete, though approximate, probabilistic description of size effect. For a detailed formulation and justification of the nonlocal generalization of Weibull theory, see Refs. [51,1,2,33,34,39].

Considering the nonlocal averaging domains in a nonlocal model of a structure to be analogous to the links of a chain, one may calculate the probability of failure of a structure as follows [51]:

$P_{\mathrm{f}}=1-\exp \left(-\int_{V}\left\langle\frac{\hat{\sigma}(\mathbf{x})}{s_{0}}\right\rangle^{m} \frac{\mathrm{d} V(x)}{V_{0}}\right)$

Here $\langle\cdot\rangle$ denotes the positive part of the argument; the superior $\wedge$ denotes nonlocal quantities; $V$, volume of structure; $m$, Weibull modulus; $s_{0}$, Weibull scaling parameter; $\sigma(\mathbf{x})$, maximum principal stress at point of coordinate vector $\mathbf{x} ; \hat{\sigma}$, nonlocal stress; and $V_{0}$ is an elementary volume of the material for which the Weibull parameters $m$ and $s_{0}$ have been experimentally identified $\left(V_{0}\right.$ is analogous to the representative volume element, RVE, but not the same). Note that $P_{1}=\left\langle\sigma(\mathbf{x}) / s_{0}\right\rangle^{m}$ represents the small-probability tail of the cumulative probability distribution of the strength of a homogeneously stressed elementary volume $V_{0}$, and function $c(\sigma)=P_{1} / V_{0}$ is the spatial concentration function of failure probability [1,41]. It will be convenient to set

$V_{0}=l^{n} \quad(n=1,2$ or 3$)$

where $l$ can be regarded as the statistical characteristic length of the material, and $n$ is the number of dimensions in which the structure is scaled. Further, it is convenient to introduce dimensionless coordinates and size-independent dimensionless variables, by setting

$\mathbf{x}=D \xi, \quad \sigma(\mathbf{x})=\sigma_{\mathrm{N}} S(\xi)$

$V=l^{n} v, \quad \mathrm{~d} V(\mathbf{x})=l^{n} \mathrm{~d} v(\boldsymbol{\xi})$

where $D$ is the size (characteristic dimension) of the structure; $\boldsymbol{\xi}$, dimensionless coordinate vector; and $\sigma_{\mathrm{N}}=$ $P / b D$, nominal strength of structure ( $P$, maximum load; $b$, width of structure). We consider geometrically similar scaled structures of different sizes $D$, for which the corresponding points have the same dimensionless coordinate $\xi$. Then

$-\ln \left(1-P_{\mathrm{f}}\right)=\left(\frac{\sigma_{\mathrm{N}}}{s_{0}}\right)^{m} \theta^{-n} \int_{v}\langle S(\boldsymbol{\xi})\rangle^{m} \mathrm{~d} v(\boldsymbol{\xi})$

where

$\theta=l / D$

$\theta$ is the relative (dimensionless) size of the nonlocal averaging volume. Eq. (5) gives the failure probability not only as a function of structure size $D$ but also as a function of the geometry (or shape) of the structure and its loading. The effect of geometry is delivered by means of function $S(\boldsymbol{\xi})$.

\section{Extension to small sizes below the energetic-statistical transition}

A look at Eq. (5) immediately reveals that it must be limited to sufficiently large structure sizes $D$ because, in practice, the structure size $D$ (or cross section size) may be less, in fact much less, than the statistical characteristic length $l$, at which the transition between the energetic and statistical size effects is centered. An equation approximately applicable for any structure size $D$ may be found by smooth asymptotic matching of the large-size behavior $(D / l \rightarrow \infty)$ given by Eq. (5) and the small-size behavior; $\sigma_{\mathrm{N}}$ is a constant (for $D / l \rightarrow 0$ ). To this effect, we replace Eq. (5) by

$-\ln \left(1-P_{\mathrm{f}}\right)=\left(\frac{\sigma_{\mathrm{N}}}{s_{0}}\right)^{m} \zeta^{-n} \int_{v}\langle S(\boldsymbol{\xi})\rangle^{m} \mathrm{~d} v(\boldsymbol{\xi})$

(error $\left.\sim \theta^{2}\right)$

where

$\zeta=\frac{l}{\kappa l+D}$

Here $\kappa$ is an empirical constant which will be discussed later. For $D / l \rightarrow \infty$, this equation is equivalent to Eq. (5) because $\zeta \rightarrow \theta$. For $D / l \rightarrow 0$, the statistical size effect disappears because $\zeta \rightarrow$ constant. This agrees with the fact that, in the limit $D \rightarrow 0$, the cohesive crack model exhibits no size effect.

As indicated in Eq. (7), the error for large sizes is expected to be $\mathrm{O}\left(\theta^{2}\right)$ because Eq. (8) captures only the first two terms of the Taylor series expansion of $\zeta$ as a function of $\theta$.

\section{Approximation of nonlocal averaging}

The nonlocal stress in Eq. (1) is defined as $E$-times the nonlocal strain $\epsilon(\mathbf{x})$, which in turn is calculated as the sum of the local elastic strain $\epsilon^{\mathrm{el}}(\mathbf{x})$ and the spatially averaged (nonlocal) inelastic strain $\hat{\epsilon}^{\prime \prime}(\mathbf{x})$. However, this general definition does not allow the size effect to be described analytically. To obtain an analytical description, we first limit attention to large enough structures such that the nonlocal averaging domain, roughly of the same size as the FPZ (the zone of localized distributed cracking, or localized damage), is small compared to $D$. In that case, the FPZ size is approximately constant (independent of $D$ ).

The nonlocal stress $\hat{\sigma}$ within the zone of localized damage (distributed cracking) may be assumed to be 
approximately uniformly distributed and equal to the elastically calculated stress that existed at the center of this zone before the stresses have redistributed due to damage [6,1]. Approximately, for most practical purposes

$$
\hat{\sigma} \approx \sigma_{\mathrm{N}}\left(S_{\max }-S^{\prime} \frac{\gamma l}{D}\right) \quad\left(\text { error } \sim \theta^{2}\right)
$$

where $S_{\max }$ is the maximum dimensionless stress in the structure before cracking damage occurs (i.e. the elastically calculated stress, as in classical Weibull theory); $S^{\prime}$ is the magnitude of the dimensionless stress gradient at the maximum stress point in the direction toward the center of the FPZ (a zone representing the region of localized damage or distributed cracking); and $\gamma$ is a geometry-dependent factor selected so that $\hat{\sigma}$ would approximately represent the stress in the middle of the FPZ; $\gamma$ is constant when geometrically similar structures are considered. The foregoing definition of $\hat{\sigma}$ is of course not acceptable for those situations where gradient $S^{\prime}$ at the maximum stress point vanishes, but such situations, which may be encountered when the maximum stress occurs inside the structure rather than on its surface, are rare.

As an example, consider an unnotched (unreinforced) beam of depth $D$ subjected at the critical cross section to bending moment $M$. One may choose $\sigma_{\mathrm{N}}=\sigma_{\max }=6 M / b D^{2}=$ elastic stress at tensile face. Then $\sigma(x)=2 \sigma_{\mathrm{N}} \xi, \xi=x / D, x$ is the cross section coordinate measured from the neutral axis, $S(\xi)=2 \xi ; S^{\prime}=\mathrm{d} S /$ $\mathrm{d} \xi=2 / D$ and $\gamma=1 ; 2 l$ is the thickness of the FPZ, which is here represented by a boundary layer of distributed cracking (or localized damage) at the tensile face.

Assuming the structure to be sufficiently large compared to the FPZ, we may further assume that the nonlocal stress to be used in the failure probability integral for FPZ (i.e. the region of localized damage) is approximately uniform throughout the FPZ and equal to $\hat{\sigma}$ as given by Eq. (9). This is the simplest way to capture the effect of stress redistribution (and the corresponding energy release) caused by the formation of the FPZ. Based on this simplifying idea, the integration in Eq. (5) is possible and can be subdivided into two domains, domain $\mathscr{Z}$ of the FPZ and domain $\mathscr{R}$ of the rest of the structure volume. Eq. (5) may then be written as

$$
\begin{aligned}
-\ln \left(1-P_{\mathrm{f}}\right)\left(\frac{s_{0}}{\sigma_{\mathrm{N}}}\right)^{m} \zeta^{n}= & \int_{\mathscr{Z}}\left\langle S_{\mathrm{max}}-S^{\prime} \frac{\gamma l}{D}\right\rangle^{m} \mathrm{~d} v(\boldsymbol{\xi}) \\
& +\int_{\mathscr{R}}\langle S(\boldsymbol{\xi})\rangle^{m} \mathrm{~d} v(\boldsymbol{\xi}) \\
-\ln \left(1-P_{\mathrm{f}}\right)\left(\frac{s_{0}}{\sigma_{\mathrm{N}}}\right)^{m} \zeta^{n}= & \left\langle S_{\max }-S^{\prime} \frac{\gamma l}{D}\right\rangle^{m} v_{\mathscr{Z}}+S_{\mathscr{R}}^{m} \\
& \left(\text { error } \sim \theta^{2}\right)
\end{aligned}
$$

where

$v_{\mathscr{Z}}=\int_{\mathscr{Z}} \mathrm{d} v(\boldsymbol{\xi}), \quad S_{\mathscr{R}}^{m}=\int_{\mathscr{R}}\langle S(\boldsymbol{\xi})\rangle^{m} \mathrm{~d} v(\boldsymbol{\xi})$

Here we denoted by $v_{\mathscr{Z}}$ the volume of the FPZ in dimensionless coordinates for size $D=l$, and took into account the fact that the actual volume of the FPZ, which is $V_{\mathrm{PFZ}}=(D / l)^{n} v_{\mathscr{Z}}$, is approximately constant, independent of structure size $D$.

The approximations in Eqs. (10) and (11) are valid only for large enough structures, $D \gg \gamma l$. In terms of a power series expansion in $\theta$, they are accurate up to the linear term $\theta$, i.e., have an error $\mathrm{O}\left(\theta^{2}\right)$ It will be convenient to consider also very small structure. It is again simpler to do that asymptotically, for hypothetical structures so small that $D<\gamma l$. In that case, the point at distance $\gamma l$ from the point of $S_{\max }$ would lie outside structure, and so the asymptotic behavior for $D / l \rightarrow 0$ would appear meaningless. We may remedy it by replacing $S^{\prime} \gamma l / D$ on the right-hand sides of the foregoing two equations by $S^{\prime} \gamma \eta$ where

$\eta=\frac{l}{\gamma l+D}$

which has the asymptotic behaviors $\eta=l / D$ for $D / l \rightarrow \infty$ and $\eta$ is constant for $D / l \rightarrow 0$. Thus we get the following approximation, applicable through the entire size range:

$$
\begin{aligned}
-\ln \left(1-P_{\mathrm{f}}\right)\left(\frac{s_{0}}{\sigma_{\mathrm{N}}}\right)^{m} \zeta^{n}= & \left\langle S_{\max }-S^{\prime} \gamma \eta\right\rangle^{m} v_{\mathscr{Z}}+S_{\mathscr{R}}^{m} \\
& \left(\text { error } \sim \theta^{2}\right)
\end{aligned}
$$

It may be noted that, in considering the case $D \ll l$, it will be more appropriate to replace the nonlocal model by the cohesive crack model. Both are approximately equivalent if the damage localizes into a band, but the latter can be theoretically extended to arbitrarily small sizes $D$, much smaller than the inhomogeneity size of the actual material. Although such an extension is a fiction, it is useful for asymptotic matching.

\section{Intermediate asymptotics}

Fitting the existing experimental data (Fig. 1) for concrete [39] and laminates [42] showed that the transition from energetic (nonlocal) size effect to statistical size effect is centered at a beam depth of about $2 \mathrm{~m}$ (i.e. the size effect in flexural strength, or modulus of rupture, of laboratory beams is mainly energetic, or nonlocal, while the size effect on flexural strength of an arch dam is mainly statistical). Therefore, $\kappa l$ for concrete is of the order of meters, i.e. about two orders of magnitude larger than the aggregate size. Likewise for sea ice (Bažant, 2000), assuming the same ratio of the inhomogeneity size. On the other hand, for fine grained ceramics, $\kappa l$ would be of the order of millimeters, and for the flexural strength of fiber reinforced polymer 

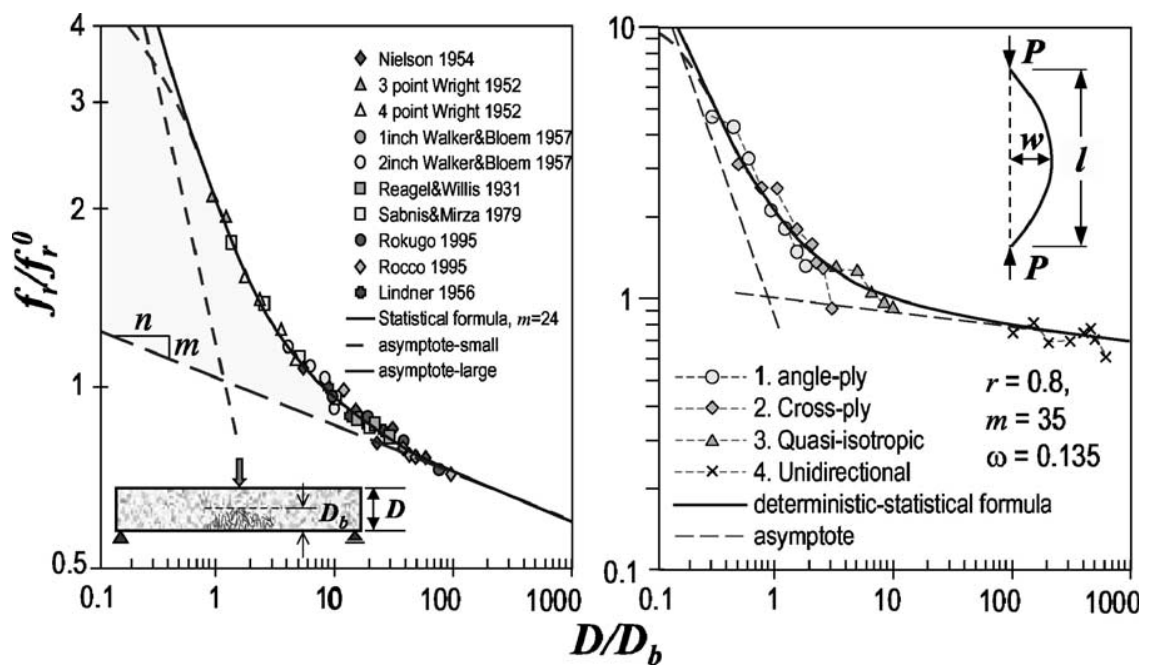

Fig. 1. Dimensionless size effect curve for modulus of rupture $f_{\mathrm{r}}$ (flexural strength) obtained after the values of $D_{\mathrm{b}}$ and $f_{\mathrm{r}}^{0}=s_{0} f_{0}$, different for each data set, have been identified by separate fitting of Eq. (30) to each set (the dashed curve is the deviation of Eq. (32). Left: concretes (see Ref. [39], giving data sources). Right: fiber-composite laminates (see Ref. [40]; data from Ref. [43,44]).

composites of the order of decimeters [42] (for a jointed rock mass, with joints about $10 \mathrm{~m}$ apart, $\kappa$ ) would be about $1 \mathrm{~km}$; for the ice cover of the Arctic Ocean as a whole, consisting of weakly connected thick mile-size floes, $\kappa l$ would probably be about 100 miles).

In contrast, the size of the nonlocal averaging zone $l$ (half the FPZ size) for concrete is about 2-3 maximum aggregate sizes, i.e. about two orders of magnitude smaller, which means that

$\kappa \approx 100, \quad \gamma \approx 1$

Generally, $l$ may be expected to be of the same order of magnitude as the inhomogeneity size in the material $(10 \mu \mathrm{m}$ for fine-grained ceramics, a few milimeters for laminates, $30 \mathrm{~m}$ for jointed rock, a few kilometers for Arctic Ocean cover).

Consequently, there exist intermediate structure sizes $D$ which are small compared to $\gamma l$ and large compared to $l$. Therefore, as a simplification, we will postulate the existence of an (approximate) intermediate asymptotic behavior [36,37] within the size range

$\gamma l \ll D \ll \kappa l$

such that: (a) $D / \kappa l$ may be considered to approach 0 while $D$ is sufficiently larger than $\gamma l$, and (b) $D / \gamma l$ may be considered to approach $\infty$ while $D$ is sufficiently smaller than $\kappa l$. The former is considered next.

\section{Large-size asymptotics of size effect}

First we consider the large-size asymptotic behavior, i.e. $D \gg \kappa l \gg \gamma l$, in which case

$\zeta \approx l / D=\theta \rightarrow 0, \quad \eta \approx l / D=\theta \rightarrow 0$
Then Eq. (14) becomes

$-\ln \left(1-P_{\mathrm{f}}\right)\left(\frac{s_{0}}{\sigma_{\mathrm{N}}}\right)^{m}\left(\frac{l}{D}\right)^{m}=S_{0}^{m}$

with constant $S_{0}$ defined as

$S_{0}=\left(v_{\mathscr{L}} S_{\max }^{m}+S_{\mathscr{R}}^{m}\right)^{1 / m}$

Hence

$\sigma_{\mathrm{N}}=\left[-\ln \left(1-P_{\mathrm{f}}\right)\right]^{1 / m} \frac{s_{0}}{S_{0}}\left(\frac{l}{D}\right)^{n / m}$

(for $\zeta \rightarrow \infty, \quad \eta \rightarrow \infty$ )

So the large-size asymptotic behavior is the pure Weibull statistical size effect, as expected on physical grounds.

\section{Approach to intermediate asymptotics from large size}

Second, consider the approach from the large size to the intermediate asymptotics in the range (16), for which

$\zeta^{n} \approx \kappa^{-n}=$ constant,$\quad \eta \approx l / D=\theta \rightarrow 0$

From Eq. (14) we have

$-\ln \left(1-P_{\mathrm{f}}\right)\left(\frac{s_{0}}{\sigma_{\mathrm{N}}}\right)^{m} \kappa^{-n}=S_{\max }^{m}\left(1-\frac{S^{\prime}}{S_{\max }} \theta\right)^{m} v_{\mathscr{Z}}+S_{\mathscr{R}}^{m}$

$-\ln \left(1-P_{\mathrm{f}}\right)\left(\frac{s_{0}}{\sigma_{\mathrm{N}}}\right)^{m} \kappa^{-n} \approx S_{\max }^{m}\left(1-\frac{m S^{\prime}}{S_{\max }} \theta\right) v_{\mathscr{L}}+S_{\mathscr{R}}^{m}$ (error $\sim \theta^{2}$ )

$-\ln \left(1-P_{\mathrm{f}}\right)\left(\frac{s_{0}}{\sigma_{\mathrm{N}}}\right)^{m} \kappa^{-n}=S_{0}^{m}\left(1-\frac{m S^{\prime} v_{\mathscr{Z}}}{S_{0}^{m}} \theta\right)$ 


$$
\begin{aligned}
-\ln \left(1-P_{\mathrm{f}}\right)\left(\frac{s_{0}}{\sigma_{\mathrm{N}}}\right)^{m} \kappa^{-n} \approx & S_{0}^{m}\left(1+\frac{m S^{\prime} v_{\mathscr{H}}}{S_{0}^{m}} r \theta\right)^{-1 / r} \\
& \left(\text { error } \sim \theta^{2}\right)
\end{aligned}
$$

Because of the simplification made already in Eq. (9), only the first two terms of the asymptotic series expansion $\sigma_{\mathrm{N}}$ in terms of powers of $l / D$ can be expected to be realistic. Therefore, any other approximation that shares the same first two terms of this expansion is equally valid. This fact was exploited in passing from Eq. (24) to (25), in which a crucial step has been introduced. Eq. (24) would give negative values when $\theta$ is large enough (or $D$ small enough), which would preclude asymptotic matching. Eq. (25) cannot do that but is equally justified because all the foregoing expressions are accurate only up to the first two terms of the asymptotic series expansion in terms of powers $l / D$.

In the last equation, exponent $r$ was introduced for the sake of generality. This is admissible because all the expressions are only first-order accurate in $\theta$ and exponent $r$ affects only the second and higher terms of the Taylor series expansion in $\theta$.

Solving Eq. (25) for $\sigma_{\mathrm{N}}$, we find the intermediate asymptotic behavior

$\sigma_{\mathrm{N}}=\left[-\ln \left(1-P_{\mathrm{f}}\right)\right]^{1 / m} s_{0} f_{0}\left(1+r \frac{D_{\mathrm{b}}}{D}\right)^{1 / r}$

(error $\left.\sim \theta^{2}\right)$

where $f_{0}$ and $D_{\mathrm{b}}$ are constants defined as follows:

$f_{0}=1 /\left(S_{0} \kappa^{n / m}\right), \quad D_{\mathrm{b}}=l v_{\mathscr{Z}} S^{\prime} / S_{0}$

The small-size (intermediate) asymptote of Eq. (26) is

$\sigma_{\mathrm{N}} \approx\left[-\ln \left(1-P_{\mathrm{f}}\right)\right]^{1 / m} s_{0} f_{0}\left(r \frac{D_{\mathrm{b}}}{D}\right)^{1 / r} \quad\left(\right.$ for $\left.D \ll D_{\mathrm{b}}\right)$

\section{Matching the large-size and intermediate asymptotics of size effect}

Now we need to realize what are the relative magnitudes of exponents. The Weibull modulus for concrete is about $m=24$ (it used to be thought that $m=12$ but this value was recently obtained after filtering our energetic size effect [34], although it used to be thought that $m \approx 12$ ). The fracture scaling is usually considered in two dimensions, i.e. $n=2$, and so the typical value $n / m=1 / 12$. According to comparisons with test data, the empirical exponent $r$ is generally between 1 and 2. For most materials, $m$ is between 15 and 50. Therefore

$n / m \ll 1, \quad r n / m \ll 1$

A continuous transition between Eqs. (20) and (28) matching the large-size asymptotic behavior and the intermediate (mid-size) asymptotic behavior may be written as follows

$\sigma_{\mathrm{N}}=\left[-\ln \left(1-P_{\mathrm{f}}\right)\right]^{1 / m} s_{0} f_{0}\left[\left(\frac{D_{\mathrm{b}}}{D}\right)^{r n / m}+\alpha r \frac{D_{\mathrm{b}}}{D}\right]^{1 / r}$

(error $\left.\sim \theta^{2}\right)$

where $\alpha$ is a constant obtained by equating Eq. (28) to (20)

$\alpha=\left(l / D_{\mathrm{b}}\right)^{n / m} /\left(S_{0} f_{0}\right)$

Because $n / m \ll 1$, the first term in the parenthesis in Eq. (30) dominates for large sizes (small $1 / D$ ), and the second term for small sizes (large 1/D). So, indeed, Eq. (30) reduces to Eq. (28) for small sizes (in the intermediate asymptotics range) and to Eq. (30) for large sizes.

The expression on the right coincides (except for the value of coefficient $\alpha$ ) with the mean size effect previously proposed on the basis of some less fundamental arguments and verified by comparisons with extensive test data (Fig. 1) on the size effect on flexural strength (modulus of rupture) of plain concrete [39]. This equation was further shown to agree with the data from flexural strength tests of laminates of various lay-ups [40, 42] (Fig. 1) conducted at NASA Langley Research Center by Jacksom et al. [43] (see also Refs. [44-46]). The Weibull size effect formula was shown incapable of fitting these test data.

However, a purely energetic formula (representing the special case for $m \rightarrow \infty$ ) was found to agree with the flexural test data for plain concrete as well as laminates equally well. The reason probably is that the test specimens were not large enough. It appears that the statistical part of size effect can get significantly manifested only for beam depths of several meters or more, which is the case, e.g., for arch dams, massive retaining walls, foundation plinths, walls of large tanks (e.g. for liquefied natural gas), and massive lining of subway stations.

\section{Matching of zero-size and intermediate asymptotics}

Eq. (30) can match closely the existing test data for flexural strength (modulus of rupture) of concrete as well as fiber composite laminates (Fig. 1), down to the smallest specimens that can be made $[39,42]$ (i.e. up to the point where the size effect curve begins to deviates down from the intermediate asymptote in Fig. 1 or Fig. 2). However, the limiting nominal strength $\sigma_{\mathrm{N}}$ for $D \rightarrow 0$ is infinite. From a purely practical viewpoint, this might not be considered as objectionable because unreasonably large $\sigma_{\mathrm{N}}$ might be indicated only for a hypothetical structure size $D$ much smaller than the inhomogeneity size, e.g. the maximum aggregate size $d_{\mathrm{a}}$ for concrete or the thickness of a lamina in a composite. For example, it is not objected that the classical Hall-Petch formula [47] for the effect of crystal size on the yield strength of polycrystalline metals behaves in such 


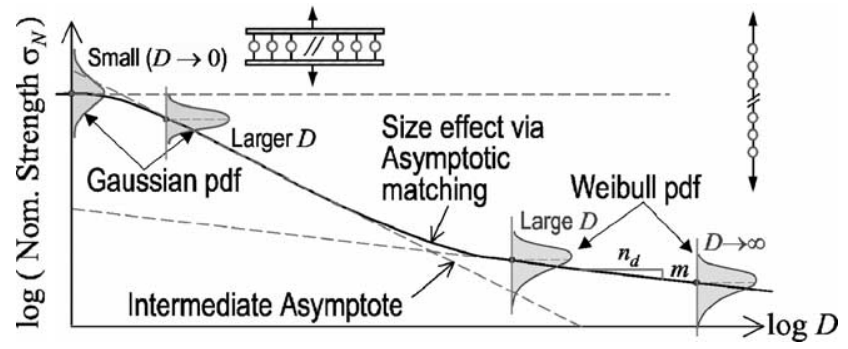

Fig. 2. The curve of mean size effect for structures failing at macroscopic fracture initiation, and its probability distributions for various sizes.

a way. Likewise, for unreinforced concrete beams in flexure, an unreasonably large $\sigma_{\mathrm{N}}$ is attained only for hypothetical beam depths smaller than $d_{\mathrm{a}}$, which is no problem for practical usage.

To make asymptotic matching approximation realistic, it is nevertheless beneficial if the small-size asymptotic properties agree with the theoretical small-size asymptotic properties of the underlying continuum model, which is the cohesive (or fictitious) crack model, or the crack band model, or the nonlocal damage model, either of which implies that the value of $\hat{\sigma}_{\mathrm{N}}$ for $D \rightarrow 0$ should be finite and should be approached linearly in $D[2,4]$. Such theoretically desired small-size asymptotic properties are needed to allow the probability distribution of size effect to be extended to the entire size range $(0, ?)$, which may be achieved by asymptotic matching of the intermediate and zero-size asymptotics. This goal was already foreseen in Eq. (13), and may be easily accomplished by applying to $D_{\mathrm{b}} / D$ in Eq. (30) the same transformation as in passing from $l / D$ to $\eta=l /(\gamma l+D)$, i.e. by replacing $D_{\mathrm{b}} / D$ with $D_{\mathrm{b}} /(\gamma l+D)$. This yields

$\sigma_{\mathrm{N}}=\left[-\ln \left(1-P_{\mathrm{f}}\right)\right]^{1 / m} s_{\mathrm{D}} \quad\left(\right.$ error $\left.\sim \theta^{2}\right)$,

where

$s_{\mathrm{D}}=s_{0} f_{0}\left[\left(\frac{D_{\mathrm{b}}}{\gamma l+D}\right)^{r n / m}+\alpha r \frac{D_{\mathrm{b}}}{\gamma l+D}\right]^{1 / r}$

If the failure probability $P_{\mathrm{f}}$ is specified, this equation gives the general approximate law of size effect for failures at crack initiation. In contrast to Eq. (30), this law gives $\sigma_{\mathrm{N}} \rightarrow$ constant for $D / \gamma l \rightarrow 0$. This is required by the cohesive crack model (as well nonlocal damage model), which was generally proven in Refs. [2,4].

Further it was proven $[2,4]$ that the zero-size limit of the cohesive crack model (in which the stress-separation curve begins with a finite slope) must be approached linearly in terms of $D / l$. This additional asymptotic property may be verified by introducing in Eq. (33) the following approximations for small $D \ll l$, having error $\mathrm{O}\left(D^{2}\right)$;

$$
\begin{aligned}
\frac{1}{(\gamma l+D)^{1 / r}} & =(\gamma l)^{-1 / r}\left(1+\frac{D}{\gamma l}\right)^{-1 / r} \\
& \approx(\gamma l)^{-1 / r}\left(1-\frac{D}{r \gamma l}\right)
\end{aligned}
$$

in which we made use of the binomial series expansion

$$
\begin{aligned}
(1+x)^{p}= & 1+p x+\frac{1}{2 !} p(p-1) x^{2}+\frac{1}{3 !} p(p-1)(p-2) x^{3} \\
& +\cdots)
\end{aligned}
$$

Note that the linear approach property would be violated if, for instance, $D_{\mathrm{b}} /(\gamma l+D)$ in Eq. (33) were replaced with $D_{\mathrm{b}}^{q} /\left(\gamma^{q l q}+D^{q}\right)$ in which $q \neq 1$.

By solving Eq. (32) for $P_{\mathrm{f}}$, we obtain the following expression for the cumulative distribution of failure probability of a structure as a function of its nominal stress $\sigma \mathrm{N}$

$P_{\mathrm{f}}=1-\mathrm{e}^{-\left(\sigma_{\mathrm{N}} / s_{\mathrm{D}}\right)^{m}} \quad\left(\right.$ error $\left.\sim \theta^{2}\right)$

where the dependence on size $D$ is introduced through function $s_{\mathrm{D}}$, given by Eq. (33).

\section{Large-size asymptotic size effect on the mean and standard deviation of $\sigma_{\mathrm{N}}$}

Similar to the classical Weibull theory, the mean of nominal strength may be calculated (with the substitution $\left.t=\left(\sigma_{\mathrm{N}} / s_{\mathrm{D}}\right)^{m}\right)$ as follows

$$
\begin{aligned}
\bar{\sigma}_{\mathrm{N}} & =\int_{0}^{1} \sigma_{\mathrm{N}} \mathrm{d} P_{\mathrm{f}}=\int_{0}^{\infty} \sigma_{\mathrm{N}} \frac{\mathrm{d} P_{\mathrm{f}}\left(\sigma_{\mathrm{N}}\right)}{\mathrm{d} \sigma_{\mathrm{N}}} \mathrm{d} \sigma_{\mathrm{N}} \\
\bar{\sigma}_{\mathrm{N}} & =\int_{0}^{\infty} \sigma_{\mathrm{N}} \mathrm{e}^{-\left(\sigma_{\mathrm{N}} / s_{\mathrm{D}}\right)^{m}} m \sigma_{\mathrm{N}}^{m-1} s_{\mathrm{D}}^{-m} \mathrm{~d} \sigma_{\mathrm{N}} \\
& =s_{\mathrm{D}} \int_{0}^{\infty} t^{1 / m} \mathrm{e}^{-t} \mathrm{~d} t \\
\bar{\sigma}_{\mathrm{N}} & =s_{\mathrm{D}} \Gamma\left(1+\frac{1}{m}\right) \quad\left(\text { error } \sim \theta^{2}\right)
\end{aligned}
$$

where $s_{\mathrm{D}}$ depends on the size, $D$. The standard deviation of $\sigma_{\mathrm{N}}$, denoted as $\delta_{\mathrm{N}}$, is given by the following calculation

$$
\begin{aligned}
\delta_{\mathrm{N}}^{2} & =\int_{0}^{1}\left(\sigma_{\mathrm{N}}-\bar{\sigma}_{\mathrm{N}}\right)^{2} \mathrm{~d} P_{\mathrm{f}}=\int_{0}^{1} \sigma_{\mathrm{N}}^{2} \mathrm{~d} P_{\mathrm{f}}-\bar{\sigma}_{\mathrm{N}}^{2} \\
\delta_{\mathrm{N}}^{2} & =\int_{0}^{\infty} m\left(\sigma_{\mathrm{N}} / s_{\mathrm{D}}\right)^{m+1} \mathrm{e}^{-\left(\sigma_{\mathrm{N}} / s_{\mathrm{D}}\right)^{m}} \mathrm{~d} \sigma_{\mathrm{N}}-\bar{\sigma}_{\mathrm{N}}^{2} \\
& =s_{\mathrm{D}}^{2} \int_{0}^{\infty} t^{2 / m} \mathrm{e}^{-t} \mathrm{~d} t-\bar{\sigma}_{\mathrm{N}}^{2} \\
\delta_{\mathrm{N}}^{2} & =s_{\mathrm{D}}^{2} \Gamma\left(1+\frac{2}{m}\right)-\bar{\sigma}_{\mathrm{N}}^{2} \quad\left(\text { error } \sim \theta^{2}\right)
\end{aligned}
$$

According to the foregoing result, the coefficient of variation of $\sigma_{\mathrm{N}}$ is

$$
\omega_{\mathrm{N}}=\frac{\delta_{\mathrm{N}}}{{\overline{\sigma_{\mathrm{N}}}}}=\sqrt{\frac{\Gamma(1+2 / m)}{\Gamma^{2}(1+1 / m)}-1} \quad\left(\text { error } \sim \theta^{2}\right)
$$

It is noteworthy that, according to the large size asymptotic approximations that we have made, the coefficient of variation of the nominal strength $\sigma_{\mathrm{N}}$ is independent of 
structure size $D$, and is given by the same expression as in Weibull theory. This means that, for large sizes, Weibull modulus $m$ can be identified from the experimentally observed scatter of the test results on identical specimens of one size. The $m$ value identified from scatter must be the same as the $m$ value identified from the size effect tests. When both results for $m$ are not equal, or when $\omega$ is found to depend on $D$, it is an indication that Weibull statistical theory does not apply, and this indeed happens for small specimens of quasibrittle materials. Such discrepancies, the checks for which have been missing from most previous experimental studies, represent a further confirmation that Weibull distribution must gradually change to some other distribution as the structure size is reduced. This question will be pursued next.

\section{Transition of statistical distribution between small and large sizes}

Now it must be realized that the Weibull statistical distribution in Eq. (35), as well as the statistics in Eqs. (38), (41) and (42), have (as marked) an error of the order of $(l / D)^{2}$, because they have been obtained solely on the basis of large size asymptotic analysis. Introduction of size parameter $\eta$ in Eq. (13) for the small-size range was in part justified by noting that, when the structure size is smaller than the nonlocal averaging volume, the entire structure must be treated as one material element. Thus, the failure cannot be governed by the weakest element because the whole structure effectively represents a single element. Consequently, the structure cannot be simulated by the weakest link model for a chain (unless the weakest link model could be applied to the microscopic bonds on the subscale of material microstucture).

A further refinement of the asymptotic matching is, therefore, needed to achieve a smooth transition between the small-size value of $\omega_{\mathrm{N}}$ and the large-size value of $\omega_{\mathrm{N}}$, the latter given by Eq. (42) (as a digression, it may be noted that, when a very broad size range (say, 1:100 or more) is considered, another kind of generalization, corresponding to the so-called broad-range size effect law characterized not by one fracture energy but a spectrum of fracture energies $[2,58]$ might be needed).

The reason that the size independence of the coefficient of variation of $\sigma_{\mathrm{N}}$ obtained in Eq. (42) is true only asymptotically for large structure sizes $D$ is that the derivation of the statistical distribution was contingent upon the assumption that $D \gg l$. Therefore, while Eq. (38) with function (32) gives a realistic size effect formula for the mean strength throughout the full size range $D \in(0, \infty)$, the coefficient of variation in Eq. (42) and the entire probability distribution $P_{\mathrm{f}}$ given by Eq. (35) are certainly invalid for small enough $D / l$.

A clue to the type of statistical distribution expected for the small-size limit may be obtained upon noting that a very small structure containing a cohesive crack (or crack band) approaches, for $D / l \rightarrow 0$, the case of an elastic body containing a perfectly plastic cohesive crack (this was rigorously proven in Ref. [2]). As is well known from the theory of plasticity, e.g. Ref. [48], failure of such a body proceeds according to a single-degree-of-freedom mechanism, in other words, is simultaneous, non-propagating, and obviously not describable by a chain model. So, for $D \rightarrow 0$, the failure probability distribution should obey Daniels' [22] 'fiber-bundle' (parallel coupling) model rather than extreme value statistics of a chain model.

But what are the material elements corresponding to the fibers in the fiber bundle model for $D \rightarrow 0$ ? The answer to this question requires a multi-scale concept of failure mechanism. From the fitting of energetic size effect to extensive experimental data for quasibrittle fracture of unreinforced concrete beams [33,34] or fiber-composite laminate strips [42], it is known that the size effect asymptote for $D \rightarrow 0$ is closely approached for extrapolation to specimen sizes smaller than the representative volume of the material. That volume spans at least three aggregate sizes in concrete.

Therefore, unlike the case of a chain model for a very large structure, the material elements corresponding to the fibers in the fiber bundle model cannot represent the representative volumes. One must reach one or two scales lower-the elements in the fiber bundle model must correspond to microscopic bonds along the failure surface in the material. These bonds, embedded in a random microstructure, must have random strength properties (again approximately described by Weibull distribution) and, as reasoned above, must be breaking along the entire failure surface nearly simultaneously, in the sense of some single-degree of freedom mechanism, i.e. there is no propagating failure front. It is in this sense that the fiber bundle model is invoked for $D \rightarrow 0$.

By formulating and solving a recursive relation for the probability distribution, Daniels [22] showed that, for a fiber bundle model whose fibers have arbitrary but identical and statistically independent probability distributions of strength (and identical elastic properties), the distribution of the nominal strength of the fiber bundle converges for increasing number $N$ of fibers to the Gaussian distribution with a mean that is asymptotically independent of $N$ and has a coefficient of variation that decreases with $N$ as $1 / \sqrt{N}$ (this is the opposite of the weakest link model, for which the mean nominal strength is known to be a power function $N^{-n / m}$ of size $N$ and the coefficient of variation to be sizeindependent, as we have confirmed by our preceding calculations.)

In our problem, $N$ is analogous to structure size $D$. Therefore, according to the fiber bundle model, the size effect on mean $\sigma_{\mathrm{N}}$ should asymptotically vanish for $D \rightarrow 0$ and the coefficient of variation of $\sigma_{\mathrm{N}}$ should asymptotically decrease as $1 / \sqrt{D}$. However, this decrease may well get offset, and perhaps even canceled, by a simultaneous 
gradual emergence of weakest-link, chain-like, behavior as size $D$ is increased.

The mean energetic size effect in concrete (as well as laminates) approaches, for decreasing $D$, the small-size plasticity asymptote. But a close approach probably normally occurs only for specimen sizes smaller that the inhomogeneity size (i.e. aggregate size in concrete). Such sizes, of course, are below the range of experiments, and so the small-size asymptotic properties are an abstract extrapolation, not directly verifiable by experiment.

The situation is similar to that already discussed with regard to the deterministic energetic size effect for concrete beams. The deterministic cohesive crack model (which is virtually equivalent to the deterministic nonlocal continuum damage model or crack band model) is widely accepted as valid, and is verified by all the test data. It has been generally proven that the small-size asymptotic limit of the cohesive crack model corresponds to an elastic body completely intersected by a perfectly plastic crack [2], but this limit is closely approached only for extrapolation to beam depth less than about $10 \%$ of aggregate size. Yet, despite this abstraction, knowledge of this small-size asymptotic limit has proven very useful for anchoring an asymptotic matching formula for the deterministic size effect, applicable in the range of experiments and real structures. It is by virtue of this precedent that we accept, for the purpose of statistics, the usefulness of a similar asymptotic limit that can be closely approached only below the practically meaningful size range.

\section{Approximate size-dependence of failure probability distribution}

Having decided the proper forms of both asymptotic probability distributions, we need to determine, at least approximately, the form of the probability distribution for finite sizes $D$. This can be most easily done by constructing an approximate asymptotic matching of these two distributions. To do this, we must be guided by the underlying deterministic size effect function $s_{\mathrm{D}}$ given by Eq. (32), but must consider the Weibull distribution in Eq. (35) to be applicable only for $D \rightarrow \infty$. For this purpose, let us write the inverse of the cumulative probability distribution in the form:

$\sigma_{\mathrm{N}}\left(P_{\mathrm{f}}\right)=\bar{\sigma}_{\mathrm{N}}(D) \Phi\left(P_{\mathrm{f}}, D\right)$

For large sizes, function $\Phi$ must agree with the inverse of Eq. (35), i.e.

$\Phi\left(P_{\mathrm{f}}, D\right)=\Phi_{\mathrm{W}}\left(P_{\mathrm{f}}\right)=\left[-\ln \left(1-P_{\mathrm{f}}\right)\right]^{1 / m} \quad(D / l \rightarrow \infty)$

where the subscript W stands for 'Weibull'. The inverse of the Gaussian distribution for the small size limit may be written as

$\Phi\left(P_{\mathrm{f}}, D\right)=\Phi_{\mathrm{G}}\left(P_{\mathrm{f}}, D\right)=1+\Psi^{-1}\left(P_{\mathrm{f}}\right) \sqrt{D / \chi l}$ where subscript $\mathrm{G}$ stands for 'Gaussian'; $\Psi^{-1}\left(P_{\mathrm{f}}\right)$ is the inverse of the unit cumulative Gaussian distribution, the mean of which is zero and standard deviation is 1 ; and $\chi$ is a dimensionless empirical constant of the order of 1. A simple formula for asymptotic matching (or smooth 'interpolation') between these two cumulative distributions may be written as follows (Fig. 2):

$\Phi\left(P_{\mathrm{f}}, D\right)=\frac{(\eta l)^{u} \Phi_{\mathrm{G}}\left(P_{\mathrm{f}}, D\right)+D^{u} \Phi_{\mathrm{W}}\left(P_{\mathrm{f}}\right)}{(\eta l)^{u}+D^{u}}$

where $u$ is an empirical constant of the order of 1 (the value $u=1 / 2$ would seem logical since it would exactly cancel the size dependence of the coefficient of variation of Daniels' fiber bundle model for small sizes). This formula gives a complete probability structure of the energetic-statistical size effect for any structure size.

Exponent $u$ controls how fast the Weibull distribution is changing to Gaussian as $D$ is decreased. Because the error of the preceding approximations is quadratic in $l / D$, the Weibull distribution probably dominates down to rather small sizes and the transition to Gaussian distribution probably happens only at very small sizes. Such behavior may be obtained by choosing $u$ sufficiently smaller than 1 .

The transition from Weibull to Gaussian distribution at diminishing structure size might explain why the traditional interpretations of test results based on coefficient of variation of random scatter of strength of identical specimens give widely different values of Weibull modulus $m$ for different specimen sizes and shapes (this fact transpires, e.g. from Bažant et al. [42] study of the data of Jackson [43] and Johnson et al. [44], for laminates, or by comparing the results of Bažant and Novák [39] with those of Zech and Wittmann [49], for concrete).

\section{Closing comments}

It is now widely accepted that the design of very large structures made of quasibrittle materials, including fiber composites or particulate composite, must take into account the size effect on structural strength. The knowledge of the mean size effect has recently advanced considerably, but the probabilistic distribution of size effect represents a glaring gap of knowledge. The present paper has outlined a mathematical approach toward closing this gap.

To a limited extent, the nonlocality of damage causing the quasibrittle mature of failure has a similar effect on the failure probability of a structure as the hypothesis of load sharing in the weakest link model of failure. However, the fracture mechanics aspects, particularly the dependence of structural failure probability on the stress redistribution due to a large FPZ or a large crack formed before failure can hardly be adequately captured by load-sharing hypotheses. In other words, expanding the existing sophisticated probabilistic models for minimum strength of seriesparallel systems in the direction of quasibrittle fracture 
mechanics with energetic size effect appears to be an extremely difficult proposition.

It therefore, appears preferable to approach the problem from the opposite side-introduce probabilistic concepts into the quasibrittle (or cohesive) fracture mechanics, and particularly the associated, recently clarified, deterministic energetic size effect model. A way to do that has been proposed in this paper, while verification by Monte Carlo finite element simulations and comparisons with test data need to be postponed for subsequent study.

\section{Acknowledgement}

Financial support Grant ONR-N00014-02-I-0622 from Office of Naval Research (monitored by Dr. Yapa D.S. Rajapakse) is gratefully acknowledged.

\section{Appendix A}

\section{Continuous transition between series and parallel coupling models}

Eq. (46) giving the continuous transition between Weibull's weakest link model and Daniel's parallel bundle model is purely phenomenological, based on no physical model. It may be more realistic to derive this transition from a physical model simulating a continuous transition between a chain and a fiber bundle. Two such models are depicted in Fig. A1(a) in which $\nu$ elements are grouped in $n$ parallel chains with $N=\nu / n$ elements in each chain; $\nu$ is very large, and $N$, varying from 1 to $\nu$, may be considered as analogous to structure size $D$.

If the distribution of elemental strength is assumed to be Weibull, $P_{\mathrm{f}}=1-\mathrm{e}^{-\left(\sigma_{\mathrm{N}} / s_{\mathrm{D}}\right)^{m}}$, then the distribution of the strength $\sigma$ of each chain of $N$ elements is also Weibull, having the form

$\left[P_{\mathrm{f}}\right]_{\text {chain }}=1-\mathrm{e}^{-N\left(\sigma / s_{\mathrm{D}}\right)^{m}}=1-\mathrm{e}^{-\left(\sigma / s_{\mathrm{DN}}\right)^{m}}$

where

$s_{\mathrm{DN}}=s_{\mathrm{D}} / N^{1 / m}$

The only difference of Eq. (A1) from the elemental distribution is that the scaling parameter $s_{\mathrm{D}}$ has changed to $s_{\mathrm{DN}}$ (more generally, the distribution for each chain at large $N$ will tend to Weibull if and only if an arbitrary elemental distribution has a power-law tail with a threshold, as required by von Mises convergence criterion [52, Eq. (4.82)], and by Fisher and Tippett's [13] stability postulate of extreme value distributions [20]. Consequently, the model in Fig. A1(a) is equivalent to a parallel coupling (or a bundle) of $n$ elements, each of which has Weibull distribution of strength given by Eq. (A1). Daniels' [22] solution of the strength of a parallel coupling (a fiber
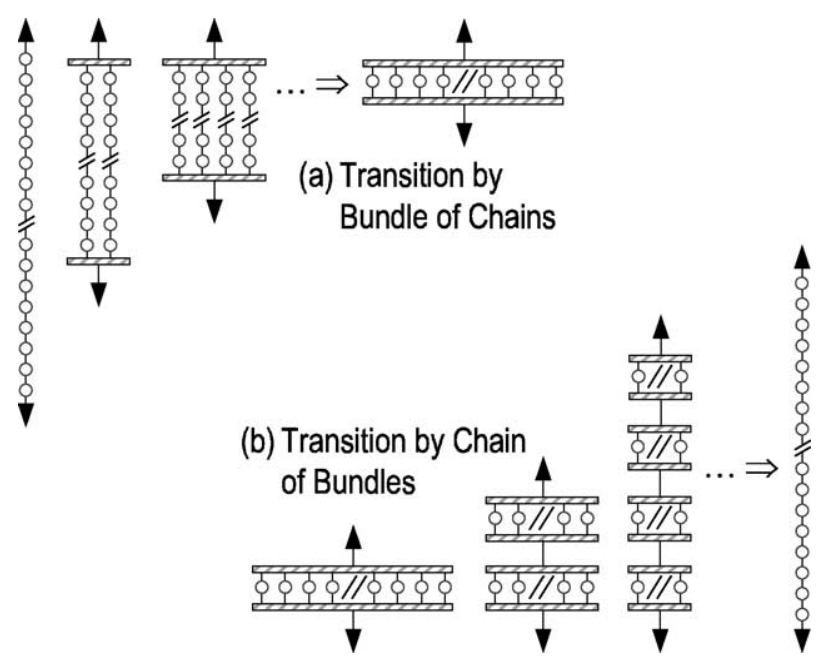

Fig. A1. (a) Bundle of chains and (b) chain of bundles as models for probability distribution describing a continuous transition between Weibull and Gaussian distributions.

bundle) may be directly applied to this parallel coupling to obtain the probability of strength of the model in Fig. A1(a). With increasing $n$, Daniels' solution converges to a Gaussian distribution with a constant mean and a coefficient of variation proportional to $1 / \sqrt{n}$.

The convergence of a system of parallel Weibull elements to the Gaussian distribution is rather slow in the far-off tail, lying beyond a certain distance $\delta_{\mathrm{G}}$ from the mean. By analogy with the central limit theorem, for a sum of $n$ random elemental variables, $\delta_{\mathrm{G}}>\delta_{\mathrm{N}} \sqrt{n}$ (although normally the Gaussian core of the distribution is broader, $\delta_{\mathrm{G}} \approx \delta_{\mathrm{N}} n^{3 / 4}$ ); however, if the elemental variables have a power-law tail (as is the case for Weibull distribution), then $\delta_{\mathrm{G}} \approx \delta_{\mathrm{N}} \sqrt{n(1+\log n)}$, which is not much larger than $\delta_{\mathrm{N}} \sqrt{n}$ [53]. Thus, we may expect the limiting Daniels-type probability distribution to be relevant to the safety factor only as long as the failure probability corresponding to the safety factor is not so small that it would lie outside the aforementioned Gaussian core.

Another simple physical model that may come to mind is shown in Fig. A1(b), in which $N$ systems of $n$ parallel elements are coupled in series. In this case, the statistics of each parallel system is simple if the strength of each element has a Gaussian distribution. Then, according to Daniels' [22] solution, the Gaussian distribution is replicated for each parallel system (with the same mean and a standard deviation decreasing as $1 / \sqrt{n}$ ). The whole system is then equivalent to a chain in which each element exhibits a Gaussian distribution. However, according to von Mises' criterion [52, Eq. (4.78)], the strength of such a chain model converges to Gumbel distribution rather than Weibull distribution. This would complicate modeling for large quasibrittle structures, and so this second alternative model is mathematically less appealing. On the other hand, this alternative might allow more realistic simulation of 
structures of positive geometry, for which the failure of one region (simulated by one parallel system) suffices to cause failure of the entire structure. Currently, this model is being developed by Pang at Northwestern University.

\section{Appendix B}

\section{How far into the tail must the probability distribution be known?}

For the purpose of design in structural or mechanical engineering, we want to achieve an extremely small failure probability $p_{\mathrm{F}}$ such as $10^{-7}$ under given random loading (which for instance means that if we would build ten million identical ship hulls or bridge girders, one and only one would fail). Estimation of loads giving such a tiny failure probability cannot be verified by properly planned experiments and thus is highly problematic. Nevertheless, we must keep in mind that if the probability structure of the problem does not have the correct form, the far-off probability tail is surely wrong. Ensuring the correct probability structure of strength and size effect, of course, does not guarantee the far-off tail to be right, but at least gives the possibility that it may be right.

If we take into account the randomness of load, then the probability $p_{\mathrm{F}}$ that the random structural resistance $R$ is less than the random load $S$ is dominated by $P_{\mathrm{f}}$ values that need not be so low. As is well known from the theory or reliability [52,54,57], the failure probability of structure under random load is given by the integral

$p_{\mathrm{F}}=\operatorname{Prob}(R<S)=\int_{0}^{\infty} f_{\mathrm{S}}\left(\sigma_{\mathrm{N}}\right) P_{\mathrm{f}}\left(\sigma_{\mathrm{N}}\right) \mathrm{d} \sigma_{\mathrm{N}}$

where $f_{\mathrm{S}}\left(\sigma_{\mathrm{N}}\right)$ is the probability density function of load described in terms of nominal stress $\sigma_{\mathrm{N}}$ as a load parameter.

For a large structure dominated by self-weight, the standard deviation of which is very small, $f_{\mathrm{S}}\left(\sigma_{\mathrm{N}}\right)$ is almost a Dirac delta function, and then the tail of $P_{\mathrm{f}}\left(\sigma_{\mathrm{N}}\right)$ must be known accurately up to $10^{-7}$. However, for a small structure dominated by live load, the standard deviation of which is large, the major contribution to the value of integral (A3) comes from a tail region of $P_{\mathrm{f}}\left(\sigma_{\mathrm{N}}\right)$ corresponding to a considerably higher probability, roughly $10^{-5}$, and in that case the tail need not be known accurately as far as $10^{-7}$.

The probability aspects of the design code are more complex. For example, if one examines carefully the current ACI Standard 318 and the way the code formulas were calibrated [56], one finds that, for example, the specifications for shear strength design of reinforced concrete beams without stirrups imply four distinct safety factors:

- $\mu_{\mathrm{L}}=1.6=$ live load factor (which dominates for small structures) or $\mu_{\mathrm{L}}=1.4=$ dead load factor acting alone (which dominates for very large structures, due to their very large self-weight).
- $\mu_{\mathrm{M}}=[(3000+1000) / 3000]^{1 / 2}=1.15$, provided we assume the design strength of concrete to be $f_{\mathrm{c}}^{\prime}=3000$ psi (because the mean strength of concrete must be 1000 psi higher than the design strength $f_{\mathrm{c}}^{\prime}=3000 \mathrm{psi}$, and because the shear strength is approximately proportional to $\sqrt{f_{\mathrm{c}}^{\prime}}$ rather than $f_{\mathrm{c}}^{\prime}$ );

- $\mu_{\mathrm{T}}=1 / 0.75=1.33=$ error factor of the theoretical formula (implied by the capacity reduction factor 0.75 ).

- $\mu_{\mathrm{E}} \approx 2=$ hidden factor for the experimental scatter of beam tests. This factor is not found in the code and its presence can be detected only if one compares the code formula to the experimental database for beam shear [56]; it arises from the fact that the design formula was made to pass at the lower margin of the data cloud rather than trough its centroid (which roughly corresponds to a probability cut-off of $5 \%$ or less in the distribution of shear force data).

As a result, the true overall safety factor of ACI beam shear design (i.e. the ratio of mean structural strength to mean load) is

$$
\begin{aligned}
\mu & =\mu_{\mathrm{L}} \mu_{\mathrm{M}} \mu_{\mathrm{T}} \mu_{\mathrm{E}} \\
& \approx \begin{cases}1.6 \times 1.15 \times 1.33 \times 2=5.0, & \text { for small size } D \\
1.4 \times 1.15 \times 1.33 \times 2=4.4 & \text { for large size } D\end{cases}
\end{aligned}
$$

Factors $\mu_{\mathrm{M}}, \mu_{\mathrm{T}}$ and $\mu_{\mathrm{E}}$ must be all be manifested in the cumulative probability distribution $P_{\mathrm{f}}\left(\sigma_{\mathrm{N}}\right)$ of the structural resistance in the reliability integral. Their uncertainty is characterized by certain probability density distributions $f_{\mathrm{M}}\left(\sigma_{\mathrm{N}}\right), f_{\mathrm{T}}\left(\sigma_{\mathrm{N}}\right)$ and $f_{\mathrm{E}}\left(\sigma_{\mathrm{N}}\right)$. If the mutual correlations are ignored, one can show that Eq. (A3) can be transformed to the form:

$$
\begin{aligned}
P_{\mathrm{F}}= & 1-\int_{-\infty}^{\infty} F_{\mathrm{S}}\left[\sigma_{N}\left(\mu_{\mathrm{L}}, \mu_{\mathrm{M}}, \mu_{\mathrm{T}}\right)\right] r_{L}\left(\mu_{L}\right) r_{M}\left(\mu_{M}\right) \\
& \times r_{T}\left(\mu_{T}\right) \mathrm{d} \sigma_{N}\left(\mu_{L}, \mu_{M}, \mu_{T}\right)
\end{aligned}
$$

in which the integral (a Stieltjes integral) represents the survival probability of the structure; factors $\mu_{L}, \mu_{M}, \mu_{T}$ are generalized as random variables with probability density distributions $r_{L}\left(\mu_{L}\right), r_{M}\left(\mu_{M}\right), r_{T}\left(\mu_{T}\right)$; and

$F_{\mathrm{S}}\left(\sigma_{\mathrm{N}}\right)=\int_{0}^{\sigma_{\mathrm{N}}} f_{\mathrm{S}}(s) \mathrm{d}(s)$

represents the cumulative probability distribution of the load. If the correlations are taken into account, the picture of course becomes more complex. Such simple considerations nevertheless clarify that if the failure probabilities are separated into several separate contributions corresponding to $\mu_{\mathrm{M}}, \mu_{\mathrm{T}}$ and $\mu_{\mathrm{E}}$, then a much shorter portion of the tail, reaching to much less than $10^{-7}$, needs to be known for each of them in order to evaluate the dominant contribution to the reliability integral. 


\section{References}

[1] Bažant ZP, Planas J. Fracture and size effect in concrete and other quasibrittle materials. Boca Raton, FL: CRC Press; 1998.

[2] Bažant ZP. Scaling of structural strength. London, UK: Hermes Penton Science (Kogan Page Science); 2002.

[3] Bažant ZP. Scaling of quasibrittle fracture: asymptotic analysis. Int J Fract 1997;83(1):19-40.

[4] Bažant ZP. Size effects in quasibrittle fracture: apercu of recent results. In: de Borst R, Mazars J, Pijandier-Cabot J, van Mier JGM, editors. Fracture mechanics of concrete structures (Proceedings, FraMCoS-4 International Conference, Paris). Lisse, Netherlands: Balkema; 2001. p. 651-8.

[5] Bažant ZP, Chen E-P. Scaling of structural failure. Appl Mech Rev ASME 1997;50(10):593-627.

[6] Bažant ZP, Li Z. Modulus of rupture: size effect due to fracture initiation in boundary layer. J Struct Engng ASCE 1995;121(4): 739-46.

[7] Bažant ZP, Li Z. Zero-brittleness size-effect method for one-size fracture test of concrete. J Engng Mech ASCE 1996;122(5): 458-68.

[8] Bažant ZP. Size effect in tensile and compression fracture of concrete structures: computational modeling and design. In: Mihashi $\mathrm{H}$, Rokugo K, editors. Fracture mechanics of concrete structures (Third International Conference, FraMCoS-3, Gifu, Japan). Freiburg, Germany: Aedificatio Publishers; 1998. p. 1905-22.

[9] Bažant ZP. Size effect in blunt fracture: concrete, rock, metal. J Engng Mech, ASCE 1984;110:518-35.

[10] Bažant ZP, Kazemi MT. Size effect in fracture of ceramics and its use to determine fracture energy and effective process zone length. J Am Ceram Soc 1990;73(7):1841-53.

[11] Bažant ZP, Daniel IM, Li Z. Size effect and fracture characteristics of composite laminates. ASME J Engng Mater Technol 1997;118(3): 317-24.

[12] Bažant ZP, Kim J-JH, Daniel IM, Becq-Giraudon E, Zi G. Size effect on compression strength of fiber composites failing by kink band propagation. Int J Fract 1999;95:103-41.

[13] Fisher RA, Tippett LHC. Limiting forms of the frequency distribution of the largest and smallest member of a sample. Proc Camb Philosoph Soc 1928;24:180-90.

[14] Weibull W. The phenomenon of rupture in solids. Proc R Swedish Inst Engng Res (Ingenioersvetenskaps Akad. Handl.), Stockholm 1939; 153:1-55.

[15] Weibull W. A statistical representation of fatigue failures in solids. Proc R Inst Tech No. 27 1949;.

[16] Weibull W. A statistical distribution function of wide applicability. J Appl Mech ASME 1951;18.

[17] Epstein B. Statistical aspects of fracture problems. J Appl Phys 1948; 19:140-7.

[18] Freudenthal AM. Physical and statistical aspects of fatigue. In: Advance in applied mechanics, vol. 4. London: Academic Press; 1956. p. $117-57$

[19] Freudenthal AM, Gumbel EJ. On the statistical interpretation of fatigue tests. Proc R Soc (Lond) A 1953;216:309-32.

[20] Gumbel EJ. Statistics of extremes. New York: Columbia University Press; 1958

[21] Saibel E. Size effect in structural safety. Proceedings of the International Conference on Structure, Solid Mechanics and Engineering Design in Civil Engineering Materials, Southampton, Part I; 1969. p. $125-30$.

[22] Daniels HE. The statistical theory of the strength of bundles and threads. Proc R Soc Lond 1945;A183:405-35.

[23] Harlow DG, Phoenix SL. The chain-of-bundles probability model for the strength of fibrous materials. I. Analysis and conjectures. J Compos Mater 1978;12:195-214.
[24] Harlow DG, Phoenix SL. The chain-of-bundles probability model for the strength of fibrous materials. ii. A numerical study of convergence. J Compos Mater 1978;12:314-34.

[25] Smith RL, Phoenix SL. Asymptotic distributions for the failure of fibrous materials under series-parallel structure and equal loadsharing. J Appl Mech 1981;48:75-81.

[26] Smith RL. The asymptotic distribution of the strength of a seriesparallel system with equal load sharing. Ann Probab 1982;10(1): 137-71.

[27] Phoenix SL, Smith RL. A comparison of probabilistic techniques for the strength of fibrous materials under local load-sharing among fibers. Int J Solids Struct 1983;19(6):479-96.

[28] McCartney LN, Smith RL. Statistical theory of the strength of fiber bundles. J Appl Mech 1983;50:601-8.

[29] Phoenix SL. The stochastic strength and fatigue of fiber bundles. Int J Fract 1983;14:327-44.

[30] Phoenix SL, Ibnabdeljalil M, Hui C-Y. Size effects in the distribution for strength of brittle matrix fibrous composites. Int J Solids Struct 1997;34(5):545-68.

[31] Phoenix SL, Beyerlein IJ. Distribution and size scalings for strength in a one-dimensional random lattice with load redistribution to nearest and next nearest neighbors. Phys Rev E 2000;62(2): $1622-45$.

[32] Mahesh S, Phoenix SL, Beyerlein IJ. Strength distributions and size effects for 2D and 3D composites with Weibull fibers in an elastic matrix. Int J Fract 2002;115:41-85.

[33] Bažant ZP, Novák D. Probabilistic nonlocal theory for quasibrittle fracture initiation and size effect. I. Theory. J Engng Mech ASCE 2000;126(2):166-74.

[34] Bažant ZP, Novák D. Probabilistic nonlocal theory for quasibrittle fracture initiation and size effect. II. Application. J Engng Mech ASCE 2000;126(2):175-85.

[35] Bažant ZP. Probabilistic modeling of quasibrittle fracture and size effect. In: Corotis RB, editor. Proceedings of the Eighth Internationsl Conference on Structural Safety and Reliability (ICOSSAR), Newport Beach, CA. Proceedings of the Eighth Internationsl Conference on Structural Safety and Reliability (ICOSSAR), Newport Beach, CA, 2001. Lisse, The Netherlands: Swets \& Zeitinger/Balkema; 2001. p. $1-23$

[36] Barenblatt GI. Similarity, self-similarity and intermediate asymptotics. New York: Consultants Bureau; 1979.

[37] Barenblatt GI. Scaling, self-similarity, and intermediate asymptotics. Cambridge: Cambridge University Press; 1996.

[38] Bender MC, Orszag SA. Advanced mathematical methods for scientists and engineers. New York: McGraw-Hill; 1978. [chapters 9-11].

[39] Bažant ZP, Novák D. Energetic-statistical size effect in quasibrittle failure at crack initiation. ACI Mater J 2000;97(3): 381-92.

[40] Bažant ZP, Zhou Y, Novák D, Daniel IM. Size effect in fracture of sandwich structure components: foam and laminate. Proceedings of the ASME International Mechanical Engineering Congress, New York), vol. AMD-TOC. New York: American Society of Mechanical Engineers; 2001. (paper 25413), p. 1-12.

[41] Freudenthal AM. Statistical approach to brittle fracture. In: Liebowitz H, editor. Fracture, vol. 2. London: Academic Press; 1968. p. 591-619. [chapter 6].

[42] Bažant ZP, Zhou Y, Novák D, Daniel IM. Size effect on flexural strength of fiber-composite laminate. ASME J Mater Technol 2003; in press.

[43] Jackson KE. Scaling effects in the flexural response and failure of composite beams. AIAA J 1992;30(8):2099-105.

[44] Johnson DP, Morton J, Kellas S, Jackson KE. Size effect in scaled fiber composites under four-point flexural loading. AIAA J 2000; 38(6): 1047-54.

[45] Wisnom MR. The effect of specimen size on the bending strength of unidirectional carbon fiber-epoxy. Compos Struct 1991;18. 
[46] Wisnom MR, Atkinson JA. Reduction in tensile and flexural strength of unidirectional glass fiber-epoxy with increasing specimen size. Compos Struct 1997;38:405-12.

[47] Cottrell AH. Iron Steel Inst Spec Rep 1963;69:281.

[48] Jirásek M, Bažant ZP. Inelastic analysis of structures. Chichester, UK Wiley; 2002.

[49] Zech B, Wittmann FH. A complex study on the reliability assessment of the containment of a PWR. Part II. Probabilistic approach to describe the behavior of materials. In: Jaeger TA, Boley TA, editors. Transaction of the Fourth International Conference on Structural Mechanics in Reactor Technology, vol. H, J1/11. Brussels, Belgium: European Communities; 1977. p. 1-14.

[50] Bažant ZP. Energetic and statistical size effects in fiber composites and sandwich structures. A précis of recent progress. In: Hui D, editor. Proceedings of the 10th International Conference on Composites/Nano Engineering (ICCE-10). University of New Orleans; 2003. p. 869-72.

[51] Bažant ZP, Xi Y. Statistical size effect in quasibrittle structures. II. Nonlocal theory. ASCE J of Engineering Mechanics 117(11): 2623-2640.
[52] Ang AH-S, Tang WH. Probability concepts in engineering planning and design. vol. II. Decision, risk and reliability. New York: Wiley; 1984.

[53] Bouchaud J-P, Potters M. Theory of financial risks: from statistical physics to risk management. Cambridge: Cambridge University Press; 2000.

[54] Freudenthal AM, Garrelts JM, Shinozuka M. The analysis of structural safety. J Struct Div ASCE 1966;92(ST1):619-77.

[55] Weibull W. Basic aspects of fatigue. Proceedings of the Colloquium on Fatigue, Stockholm, Berlin: Springer; 1956.

[56] Bažant ZP, Yu Q. Designing against size effect on shear strength of reinforced concrete beams without stirrups. Structural engineering report No. 03-02/A466s. Northwestern University, Evanston, Illinois; 2003.

[57] Haldar A, Mahadevan S. Probability, reliability and statistical methods in engineering design. New York: Wiley; 2000.

[58] Bažant ZP, Yu Q, Zi G. Choice of standard fracture test for concrete and its statistical evaluation. Intern, J. of Fracture, (2002) 128(11):1331-1336 